05

\title{
Термокинетическая модель разрушения гетерогенных материалов и особенности ее численной реализации при воздействии высокочастотными электромагнитными полями
}

\author{
(C) М.Г. Менжулин, ${ }^{1}$ Х.Ф. Махмудов ${ }^{2, \text { Ф }}$ \\ ${ }^{1}$ Национальный минерально-сырьевой университет „Горный“, \\ 199106 Санкт-Петербург, Россия \\ ${ }^{2}$ Физико-технический институт им. А.Ф. Иофффе РАН, \\ 194021 Санкт-Петербург, Россия \\ e-mail: h.machmoudov@mail.ioffe.ru
}

(Поступило в Редакцию 30 августа 2016 г. В окончательной редакции 22 декабря 2016 г.)

Теоретическими и экспериментальными методами исследованы процессы разрушения горных пород. Указана модель развития микро- и макротрещиноватости при воздействии электромагнитных полей с помощью ВЧ электродов. Расчет полей температур и полей термоупругих напряжении позволяет исследовать развитие макротрещиноватости и установить направления их развития при объемном способе их размещения. Установлены механизмы, формирующие магистральную трещину, в областях с различной величиной растягивающих напряжений. Развитие магистральной трещины может происходить в область растягивающих напряжений, меньшей предела прочности на растяжение. Показана возможность управления процессами развития магистральной трещины за счет выбора напряжений по длине образующейся трещины и времени нагрева.

DOI: 10.21883/JTF.2017.07.44675.2024

\section{Введение}

Среди разнообразных процессов, протекающих при воздействии ВЧ электромагнитных полей на горную породу, следует выделить основные:

- различные тепловые расширения минеральных компонентов при воздействии СВЧ поля. Известно [1-11], что минеральные компоненты поглощают электромагнитную энергию быстрее, чем органические, в 2-3 раза. Следовательно, происходит неодинаковое расширение различных компонентов, вызывающее ослабевание их межфазных границ и появление трещин и разломов, что затем приводит к разрушению [12-16];

- поглощение породой ВЧ энергии и формирование источников тепла, нагрев горной породы и развитие в ней термоупругих напряжений [12,14,17-26], которые приводят к развитию трещиноватости, разупрочнению и разрушению породы [27-31]. В работах [32-43] изучены основные закономерности и управления процессом разрушения материала (угля) с помощью СВЧ поля.

Микроволновое излучение приводит к существенной модификации примесно-дефектной структуры исследуемых полупроводников [44-46]. В работах [47-50] обсуждаются механизмы трансформации дефектной подсистемы монокристаллов под действием микроволновой обработки.

Показано, что изменения, которые наблюдаются в эксперименте, связаны с атермическим действием микроволнового излучения при удельной мощности $7.5 \mathrm{~W} / \mathrm{cm}^{2}$ и в большей степени при $90 \mathrm{~W} / \mathrm{cm}^{2}$.

При конкретной реализации подхода вычислительного эксперимента необходимо учитывать ряд факторов, в значительной степени влияющих на проведение работ на каждой стадии [51-62].

Разрабатываемый подход вычислительного эксперимента заключается в том, что при некотором воздействии используется физико-математическая модель [59,63-69]. В отличие от физической среды, состояние физико-математической модели может быть проконтролировано, оценено и изменено на любой временно́й стадии процесса в любой пространственной точке.

Отметим степень разработанности численных методов, используемых при решении задач, допускающих расщепление по физическим процессам; возможности аналитических методов по получению результатов в предельных и частных случаях; наличие теоретических решений и экспериментальных результатов в частных случаях; наличие экспериментальных исходных данных по пределам изменения физических величин и т.д.

\section{Методика измерений}

Формирование температурных полей и полей напряжений исследовалось на основании вычислительного метода $[13,14,26,33,59]$. В соответствии с принципом расщепления по физическим процессам [59] алгоритм решения общей задачи естественным образом расщепляется на два этапа.

Стационарный этап включает в себя задачи нахождения в блоке скальной породы потенциала ВЧ электрического поля, создаваемого системой электродов, и нахождения поля тепловых источников. Следуя [14,59], считаем, что ВЧ электрическое поле и создаваемое им 
поле тепловых источников стационарны, устанавливаются мгновенно и не зависят от остальных физических процессов нагрева породы, развития термоупругих напряжений и разрушения, что и является условием выделения этапа.

Нестационарный этап включает в себя решение задач нагрева скальной породы найденными тепловыми источниками, развития в породе термоупругих напряжений [60-64], а также трещинообразования, разрушения и разупрочнения породы под воздействием термоупругих напряжений [65-72]. При этом подэтап нахождения полей термоупругих напряжений не содержит время в качестве непосредственного аргумента, а подэтапы нахождения температурного поля и разрушения непосредственно зависят от времени.

В соответствии с принципом расщепления по физическим процессам численное решение уравнений каждого из этапов и подэтапов может быть проведено своим методом независимо от других. Расчет потенциала ВЧ электрического поля и поля тепловых источников применительно к данной задаче был выполнен численным методом. Расхождение результатов расчета $\varphi$ и $q$ с данными аналитического решения, выполненного в $[13,59,67]$, не превышает $3 \%$ в области между электродами, что свидетельствует о работоспособности разностного метода.

\section{Решение задачи развития макротрещин в блоке скальной породы системой двух стержневых ВЧ электродов}

На основе теоретических решений общей задачи ВЧ контактного способа разрушения применительно к отбойке и дроблению негабарита с использованием

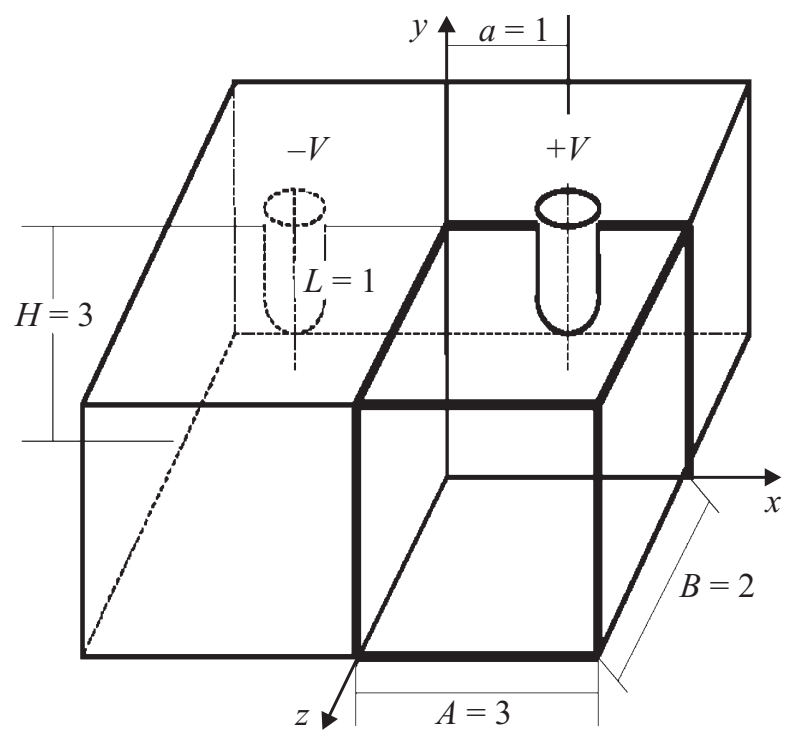

Рис. 1. Схема расчетной области в задаче о двух электродах $d / L=0.4$; сетка $15 \times 15 \times 10$ ячеек. цилиндрических электродов, направленной на изыскание новых технологий в скальных горных породах на основе использования нетрадиционных способов разрушения, решалась задача о разрушении блока системой электродов, размещенных в шпурах. Два электрода длиной $L=1$, с отношением диаметра к длине $d / L=0.4$, располагаются в шпурах на расстоянии $2 a=2$ в гранитном блоке, имеющем размеры $A=3, H=3, B=2$. К электродам приложено ВЧ напряжение $\pm V$. Вследствие симметрии задачи расчетная область составит 1/4 блока (рис. 1).

Расчетную область разбиваем сеткой на кубические ячейки $x=y=z=0.2 L$, тогда при $N_{x}=15, N_{y}=15$, $N_{z}=10$ общее число ячеек составит 2250. Учитывая выбранные размеры, ячейки, занимаемые электродом, будут $i=5-6 ; j=11-15 ; k=1$. В них задаются граничные условия, обеспечивающие $\varphi=1$ на границе электрода, $\varphi_{l+1}=2 \varphi_{l}$, где $l-$ номер расчетной ячейки, $l+1$ - номер примыкающей к ней фиктивной ячейки по соответствующей координате. Это обеспечивает автоматическое вычисление производных потенциала при расчете поля тепловых источников. Расчет полей температур и термоупругих напряжений осуществляется „сквозным“ счетом через пространственные ячейки поля без выделения границ электрода и специальной постановки на них граничных условий [26,59,65]. Граничные условия, таким образом, ставятся только на гранях блока по описанной схеме. Такая постановка задачи с точки зрения разностного счета обладает сглаживающим эффектом, что обеспечивает устойчивый счет в окрестности границ электрода.

Таким образом, получено аналитическое решение задачи определения температурного поля и поля напряжений в блоке породы при ВЧ нагреве двумя цилиндрическими электродами, расположенными в шпурах. При определении полей напряжений использована модель, не учитывающая влияния свободных поверхностей в шпуре. При этом распределения полей температур и термонапряжений показывают, что магистральная трещина будет развиваться с наружных граней блока и почти на уровне нижнего основания электрода. Второй максимум растягивающих напряжений наблюдается в верхней части блока, где также предполагается развитие магистральной трещины.

\section{Расчет поля температур в приэлектродной зоне}

Процесс нагрева блока горной породы внутренними источниками тепла, возникающими при поглощении породой энергии электрического поля, описывается уравнением нестационарной теплопроводности с граничными условиями отсутствия теплообмена на границах блока. 


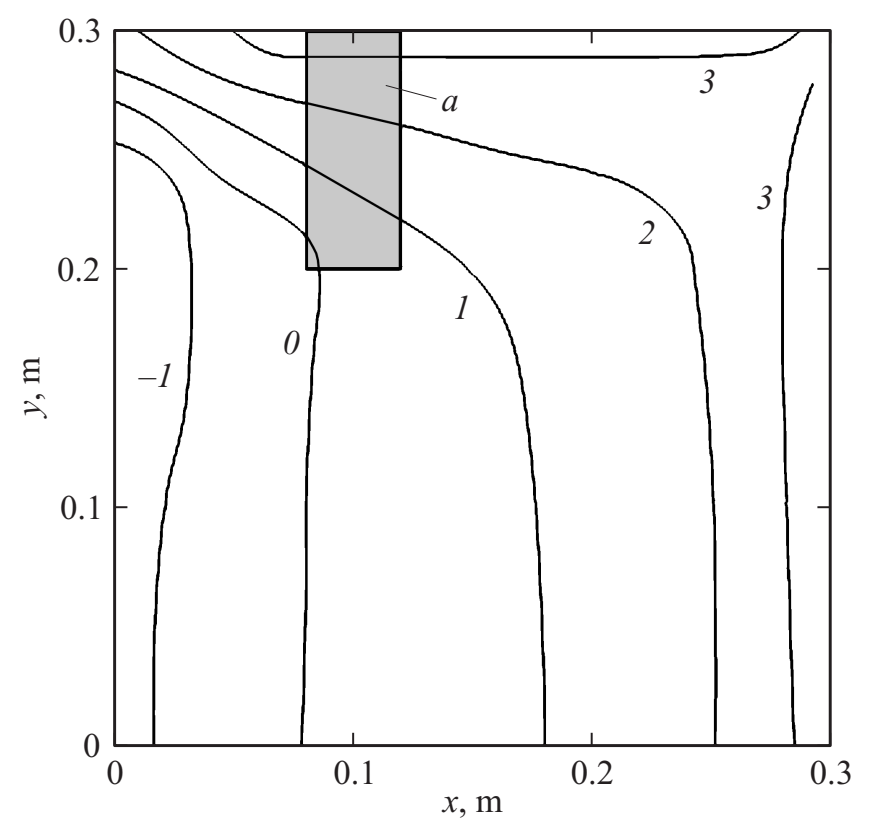

Рис. 2. Поле максимального главного напряжения $\sigma_{1}$ при $t=60 \mathrm{~s} ; z=0:-1-\sigma_{1}=55,0-\sigma_{1}=0,1-\sigma_{1}=55,2-$ $\sigma_{2}=110,3-\sigma_{1}=220 \mathrm{MPa} ; a-$ электрод.

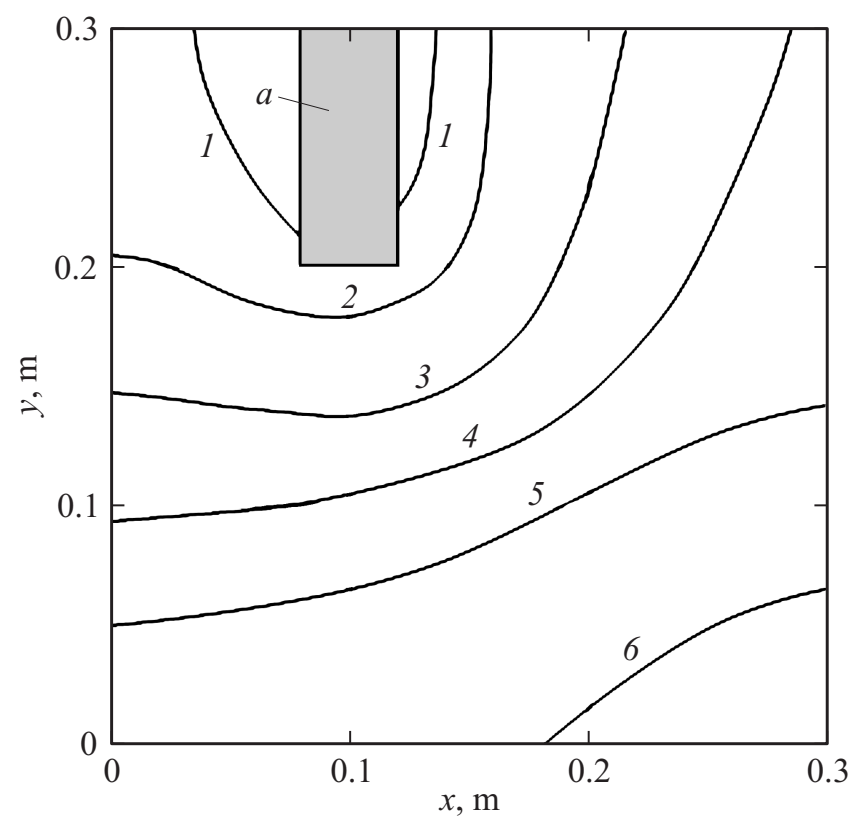

Рис. 3. Поле температуры при $t=60 \mathrm{~s} ; 1-240,2-210$, $3-180,4-170,5-160,6-150^{\circ} \mathrm{C} ; a-$ электрод.

Уравнение теплопроводности $[26,65]$

$$
c \rho \frac{\partial T}{\partial t}=a^{2}\left(\frac{\partial^{2} T}{\partial x^{2}}+\frac{\partial^{2} T}{\partial y^{2}}+\frac{\partial^{2} T}{\partial z^{2}}\right),
$$

где $c, \rho, a-$ являются постоянными величинами, можно привести к безмерному виду с помощью использования переменных: $\bar{t}=t / t_{0}, \bar{x}=x / x_{0}, \bar{y}=y / y_{0}, \bar{z}=z / z_{0}$, $\bar{q}=q / q_{0}, \quad \bar{T}=T / T_{0}, \quad \bar{\sigma}=\sigma / \sigma_{0}, q_{0}$ - объемный источник тепла, $q_{0}=\pi \varepsilon_{0} f \varepsilon^{\prime}(\operatorname{tg} \delta)|E|^{2}=\pi \varepsilon_{0} f \varepsilon^{\prime}(\operatorname{tg} \delta) V_{0}^{2} / r_{0}^{2}$, где $\varepsilon_{0}-$ диэлектрическая постоянная, $\varepsilon^{\prime}-$ диэлектрическая проницаемость породы, $\operatorname{tg} \delta$ - тангенс угла диэлектрических потерь, $f$ - частота, $E-$ напряженность электрического поля, $V-$ напряжение; $T_{0}-$ температура, $T_{0}=q_{0} r_{0}^{2} / \chi$, где $\chi-$ теплопроводность породы; $r_{0}$ - радиус электрода; $t_{0}$ - время; описывается формулой $t_{0}=C r_{0}^{2} / \chi$, где $C-$ объемная теплоемкость породы; $\sigma_{0}-$ напряжение; описывается формулой $\sigma_{0}=\alpha_{T} E T(1 /-v)$, где $E-$ модуль Юнга; $\alpha_{T}-$ коэффициент линейного расширения породы и $v-$ коэффициент Пуассона. В дальнейшем для упрощения записи черточки над переменными опускаются.

В качестве граничных условий принимается отсутствие теплообмена на границах блока [59] (рис. 2, 3)

$$
\begin{aligned}
\frac{\partial T}{\partial x}(A, y, z, t) & =\frac{\partial T}{\partial y}(x, H, z, t)=\frac{\partial T}{\partial z}(x, y, B, t)=0, \\
T(-0, y, z, t) & =T(+0, y, z, t), \\
T(x,-0, z, t) & =T(x,+0, z, t), \\
T(x, y,-0, t) & =T(x, y,+0, t)
\end{aligned}
$$

и начальное условие

$$
T(x, y, z, 0)=0 .
$$

Разностная схема $[14,59,66]$, примененная к уравнению нестационарной теплопроводности, примет вид

$$
\begin{aligned}
T_{i, j, k}^{n+1} & =\frac{1}{2\left(1+\beta_{x}^{2}+\beta_{z}^{2}\right)}\left[\beta_{x}^{2} T_{i+1, j, k}^{n}+\beta_{z}^{2} T_{i-1, j, k}^{n+1}+T_{i, j+1, k}^{n}\right. \\
& \left.+T_{i, j-1, k}^{n}+\beta_{z}^{2} T_{i, j, k+1}^{n}+\beta_{z}^{2} T_{i, j, k-1}^{n+1}+q_{i, j, k} \Delta-y^{2}\right],
\end{aligned}
$$

где $\beta x=\Delta y / \Delta x, \beta z=\Delta y / \Delta z$;

$$
\Delta t / \Delta y^{2}=1 / 2\left(1+\beta_{x}^{2}+\beta_{z}^{2}\right)
$$

$n=1, N-$ индекс текущего псевдовременного слоя; $i, j, k$ - индексы текущего временного слоя, указывающие номер счетной ячейки по соответствующей координате $x, y, z$.

Будем использовать прямоугольные расчетные области. Тогда для постановки граничных условий на границах расчетной области вводятся примыкающие к каждой из границ слои фиктивных ячеек, в которых задаются значения потенциала, обеспечивающие выполнение граничных условий. В этом случае индексы изменяются в следующих пределах: $i \in\left[0, N_{x}+1\right] ; j \in\left[0, N_{y}+1\right]$; $k \in\left[0, N_{z}+1\right]$; где $N_{x}, N_{y}, N_{z}$ - количество ячеек в расчетной области соответственно вдоль осей $x, y, z$.

Этот вид разностной схемы получен для максимально возможного временного шага, обеспечивающего устойчивость схемы [59]:

$$
\Delta t=\frac{\Delta y^{2}}{2\left(1+\beta_{x}^{2}+\beta_{z}^{2}\right)},
$$


и не может считаться удовлетворительным в общем контексте задачи о разрушении. Решение задачи нахождения поля температур является первым подэтапом нестационарного этапа, величину временного шага $\Delta t R$, на котором задается подэтап разрушения породы. При этом $\Delta t \neq \Delta t R$. Полагаем

$$
\Delta t R=m \Delta t+\Delta t^{\prime}
$$

где $m=0,1,2, \ldots$ - число максимальных тепловых шагов на одном шаге разрушения, такое, что $0 \leq \Delta t<\Delta t^{\prime}$. Для проведения расчетов разностная схема (7) должна быть представлена в виде с явным выражением для $\Delta t$ :

$$
\begin{aligned}
T_{i, j, k}^{n+1}= & T_{i, j, k}\left[1-2\left(1+\beta_{x}^{2}+\beta_{z}^{2}\right)+\frac{\Delta t}{\Delta y^{2}}\right] \\
& +\frac{\Delta t}{\Delta y^{2}}\left[\beta_{x}^{2} T_{i+1, j, k}^{n}+\beta_{x}^{2} T_{i-1, j, k}^{n+1}+T_{i, j+1, k}^{n}+T_{i, j-1, k}^{n+1}\right. \\
& \left.+\beta_{z}^{2} T_{i, j, k+1}^{n-1}+\beta_{z}^{2} T_{i, j, k-1}^{n+1}+q_{i, j, k} \Delta y^{2}\right] .
\end{aligned}
$$

В разностном виде граничные условия (3.2)-(3.5) выбранные размеры ячейки [59] (рис. 2,3) представляются следующим образом:

$$
\begin{gathered}
T_{0, j, k}^{n}=T_{1, j, k}^{n}, T_{N x+1, j, k}^{n}=T_{N x, j, k}^{n}, T_{i, 0, k}^{n}=T_{i, 1, k}^{n}, \\
T_{i, N y+1, k}^{n}=T_{i, N y, k}^{n}, T_{i, j, 0}^{n}=T_{i, j, 1}^{n}, T_{i, j, N z+1}^{n}=T_{i, j, N z}^{n} .
\end{gathered}
$$

Начальное условие (6) будет справедливо лишь на первом из шагов разрушения при $t_{R}=0$. Начальным распределением температур на каждом из последующих временны́х шагов разрушения $T^{0, l+1}$ при $t=t_{R}^{0, l+1}$, где $l$ - номер шага разрушения, будет конечное распределение температур $T^{k, l}$ на предыдущем временно́м шаге разрушения при $t=t_{R}^{k, l}$, т.е. с точки зрения температуры общий алгоритм нестационарного этапа является двуслойным. В итоге начальные условия могут быть записаны

$$
T_{i, j, k}^{0,0}=0, \quad T_{i, j, k}^{0, l+1}=T_{i, j, k}^{k, l} .
$$

Для вычисления поля температур по алгоритму (7), (11)-(13) требуется массив тепловых источников $q_{i, j, k}$ (который был получен в качестве выходного массива задачи расчета потенциала электрического поля), массив температур предыдущего временного слоя нестационарного этапа и величина временного шага $\Delta t_{R}$, получаемая из решения задачи разрушения.

\section{Расчет поля термоупругих напряжений в приэлектродной зоне}

\section{Разностный метод решения квазистационарной задачи термоупругости}

Процесс формирования в блоке скальной породы термоупругих напряжении под воздействием неоднородного температурного поля описывается уравнениями квазистационарного приближения теории термоупругости. Используя уже сформированную выше систему безразмерных переменных, систему можно преобразовать к следующему виду $[26,65]$ :

$$
\begin{aligned}
& \frac{\partial^{2} U}{\partial x^{2}}+\eta\left(\frac{\partial^{2} U}{\partial y^{2}}+\frac{\partial^{2} U}{\partial z^{2}}\right) \\
& +(1-\eta)\left(\frac{\partial^{2} V}{\partial x \partial y}+\frac{\partial^{2} W}{\partial x \partial z}\right)-\frac{\partial T}{\partial x}=0, \\
& \frac{\partial^{2} V}{\partial y^{2}}+\eta\left(\frac{\partial^{2} V}{\partial x^{2}}+\frac{\partial^{2} V}{\partial z^{2}}\right) \\
& +(1-\eta)\left(\frac{\partial^{2} U}{\partial x \partial y}+\frac{\partial^{2} W}{\partial x \partial z}\right)-\frac{\partial T}{\partial y}=0, \\
& \frac{\partial^{2} W}{\partial z^{2}}+\eta\left(\frac{\partial^{2} W}{\partial x^{2}}+\frac{\partial^{2} W}{\partial y^{2}}\right) \\
& +(1-\eta)\left(\frac{\partial^{2} U}{\partial x \partial y}+\frac{\partial^{2} V}{\partial x \partial z}\right)-\frac{\partial T}{\partial z}=0,
\end{aligned}
$$

где $U, V, W$ - компоненты вектора перемещений,

$$
\eta=\mu /(2 \mu+\lambda)
$$

где $\mu, \lambda$ - параметры Ляме [65].

Система решается методом установления с помощью разностной схемы Либмана [26]. Для этого в каждое из уравнений системы вводим нестационарные члены $\partial U / \partial t$ в уравнение (14), $\partial V / \partial t$ в уравнение $(15), \partial W / \partial t$ в уравнение (16). Введение нестационарных членов обеспечивает устойчивость системы (17). В итоге разностная схема строится следующим образом $[59,65]$ :

$$
\begin{aligned}
& U_{i, j, k}^{n+1}=U_{i, j, k}^{n} K_{U}+1 /\left(1+\beta_{x}^{2}+\beta_{z}^{2}\right) \\
& \times\left\{\beta_{x}^{2}\left(U_{i+1, j, k}^{n}+U_{i-1, j, k}^{n+1}\right)+\eta\left(U_{i, j+1, k}^{n}+U_{i, j-1, k}^{n+1}\right)\right. \\
& +\eta \beta_{z}^{2}\left(U_{i, j, k+1}^{n}+U_{i, j, k-1}^{n+1}\right)+0.25(1-\eta) \beta_{x} \\
& \times\left(V_{i+1, j, k}^{n}-V_{i-1, j, k}^{n+1}\right)\left(V_{i, j+1, k}^{n}-V_{i, j-1, k}^{n+1}\right)+0.25(1-\eta) \\
& \times \beta_{x} \beta_{z}\left(W_{i+1, j, k}^{n}-W_{i-1, j, k}^{n+1}\right)\left(W_{i, j+1, k}^{n}-W_{i, j-1, k}^{n+1}\right) \\
& \left.-0.5 \beta_{x}\left(T_{i+1, j, k}^{n}-T_{i-1, j, k}^{n+1}\right) \Delta y\right\} \Delta t
\end{aligned}
$$




$$
\begin{aligned}
& V_{i, j, k}^{n+1}=V_{i, j, k}^{n} K_{V}+1 /\left(1+\beta_{x}^{2}+\beta_{z}^{2}\right) \\
& \times\left\{\eta \beta_{x}^{2}\left(V_{i+1, j, k}^{n}+V_{i-1, j, k}^{n+1}\right)+V_{i, j+1, k}^{n}+V_{i, j-1, k}^{n+1}\right. \\
& +\eta \beta_{z}^{2}\left(V_{i, j, k+1}^{n}+V_{i, j, k-1}^{n+1}\right)+0.25(1-\eta) \beta_{x} \\
& \times\left(U_{i+1, j, k}^{n}-U_{i-1, j, k}^{n+1}\right)\left(U_{i, j+1, k}^{n}-U_{i, j-1, k}^{n+1}\right) \\
& \quad+0.25(1-\eta) \beta_{z}\left(W_{i, j+1, k}^{n}-W_{i, j-1, k}^{n+1}\right), \\
& \left.\left(W_{i, j, k+1}^{n}-W_{i, j, k-1}^{n+1}\right)-0.5 \beta_{y}\left(T_{i, j+1, k}^{n}-T_{i, j-1, k}^{n+1}\right) \Delta y\right\} \Delta t, \\
& W_{i, j, k}^{n+1}=W_{i, j, k}^{n} K_{W}+1 /\left(1+\beta_{x}^{2}+\beta_{z}^{2}\right) \\
& \times\left\{\eta \beta_{x}^{2}\left(W_{i+1, j, k}^{n}+W_{i-1, j, k}^{n+1}\right)+\eta\left(W_{i, j+1, k}^{n}+W_{i, j-1, k}^{n+1}\right)\right. \\
& +\beta_{z}^{2}\left(W_{i, j, k+1}^{n}+W_{i, j, k-1}^{n+1}\right)+0.25(1-\eta) \beta_{x} \beta_{z} \\
& \times\left(U_{i+1, j, k}^{n}-U_{i-1, j, k}^{n+1}\right)\left(U_{i, j, k+1}^{n}-U_{i, j, k-1}^{n+1}\right) \\
& +0.25(1-\eta) \beta_{z}\left(V_{i, j+1, k}^{n}-V_{i, j-1, k}^{n+1}\right)\left(V_{i, j, k+1}^{n}-V_{i, j, k-1}^{n+1}\right) \\
& \left.-0.5 \beta_{z}\left(T_{i, j, k+1}^{n}-T_{i, j, k-1}^{n+1}\right) \Delta y\right\} \Delta t,
\end{aligned}
$$

где

$$
\begin{aligned}
& K_{U}=(1-\eta)\left(1+\beta_{z}^{2}\right) /\left(1+\beta_{x}^{2}+\beta_{z}^{2}\right), \\
& K_{V}=(1-\eta)\left(1+\beta_{z}^{2}\right) /\left(1+\beta_{x}^{2}+\beta_{z}^{2}\right), \\
& K_{W}=(1-\eta)\left(1+\beta_{z}^{2}\right) /\left(1+\beta_{x}^{2}+\beta_{z}^{2}\right) .
\end{aligned}
$$

Соотношение между перемещениями, напряжениями и деформациями в этом случае в разностной форме принимают вид

$$
\begin{gathered}
\varepsilon_{x i, j, k}^{n+1}=\left(U_{i+1, j, k}^{n+1}-U_{i-1, j, k}^{n+1}\right) / \Delta x, \\
\varepsilon_{y i, j, k}^{n+1}=\left(U_{i, j+1, k}^{n+1}-U_{i, j-1, k}^{n+1}\right) / \Delta y, \\
\varepsilon_{z i, j, k}^{n+1}=\left(U_{i, j, k+1}^{n+1}-U_{i, j, k-1}^{n+1}\right) / \Delta z, \\
\varepsilon_{x z i, j, k}^{n+1}=0.5\left(V_{i, j, k+1}^{n+1}-V_{i, j, k-1}^{n+1}\right) / \Delta z \\
+0.5\left(W_{i, j+1, k}^{n+1}-W_{i, j-1, k}^{n+1}\right) / \Delta y, \\
\varepsilon_{z z i, j, k}^{n+1}=0.5\left(W_{i+1, j, k}^{n+1}-W_{i-1, j, k}^{n+1}\right) / \Delta x \\
+0.5\left(U_{i, j, k+1}^{n+1}-U_{i, j, k-1}^{n+1}\right) / \Delta z, \\
\sigma_{x, i, j, k}^{n+1}=\varepsilon_{x, i, j, k}^{n+1}+\left[v e-(1-2 v) T_{i, j, k}^{n+1} /(1-2 v)\right], \\
\sigma_{y, i, j, k}^{n+1}=\varepsilon_{y, i, j, k}^{n+1}+\left[v e-(1-2 v) T_{i, j, k}^{n+1} /(1-2 v)\right], \\
\sigma_{z, i, j, k}^{n+1}=\varepsilon_{z, i, j, k}^{n+1}+\left[v e-(1-2 v) T_{i, j, k}^{n+1} /(1-2 v)\right]
\end{gathered}
$$

$$
\begin{gathered}
\sigma_{x y, i, j, k}^{n+1}=\varepsilon_{x y, i, j, k}^{n+1}, \\
\sigma_{y z, i, j, k}^{n+1}=\varepsilon_{y z, i, j, k}^{n+1}, \\
\sigma_{z x, i, j, k}^{n+1}=\varepsilon_{z x, i, j, k}^{n+1}, \\
e=\varepsilon_{x i, j, k}^{n+1}+\varepsilon_{y i, j, k}^{n+1}+\varepsilon_{z i, j, k}^{n+1},
\end{gathered}
$$

где $\beta x, \beta z, \Delta t-$ вычисляются в соответствии с (3).

Граничные условия для квазистационарной задачи термоупругости заданы условиями свободного перемещения граней блока и отсутствием нормальных перемещений в плоскостях симметрии:

$$
\begin{aligned}
& \partial U / \partial x(A, y, z)=0, \quad \partial V / \partial x(A, y, z)=0, \\
& \partial W / \partial x(A, y, z)=0, \quad \partial U / \partial y(x, a, z)=0, \\
& \partial V / \partial y(x, a, z)=0, \quad \partial W / \partial y(x, a, z)=0, \\
& \partial U / \partial z(x, y, B)=0, \quad \partial V / \partial z(x, y, B)=0, \\
& \partial W / \partial x(x, y, B)=0, \\
& U(0, y, z)=0, \quad V(0, y, z)=0, \quad W(0, y, z), \\
& U(x, 0, z)=0, \quad V(x, 0, z)=0, \quad W(x, 0, z), \\
& U(x, y, 0)=0, \quad V(x, y, 0)=0, \quad W(x, y, 0) .
\end{aligned}
$$

В разностном виде эти граничные условия примут вид

$$
\begin{gathered}
U_{0, j, k}=-U_{1, j, k}, \quad V_{0, j, k}=-V_{1, j, k}, \quad W_{0, j, k}=-W_{1, j, k}, \\
U_{i, 0, k}=-U_{i, l, k}, \quad V_{i, 0, k}=-V_{i, l, k}, \quad W_{i, 0, k}=-W_{i, l, k}, \\
U_{i, j, 0}=-U_{i, j, l}, \quad V_{i, j, 0}=-V_{i, j, l}, \quad W_{i, j, 0}=-W_{i, j, l},
\end{gathered}
$$$$
U_{N x+1, j, k}=U_{N x, j, k}, \quad V_{N x+1, j, k}=V_{N x, j, k},
$$$$
W_{N x+1, j, k}=W_{N x, j, k}, \quad U_{i, N y+1, k}=U_{i, N y, k},
$$$$
V_{i, N y+1, k}=V_{i, N y, k}, \quad W_{i, N y+1, k}=W_{i, N y, k},
$$$$
U_{i, j, N z+1}=U_{i, j, N z}, \quad V_{i, j, N z+1}=V_{i, j, N z},
$$$$
W_{i, j, N z}=W_{i, j, N z} .
$$

Эти граничные условия дополняются граничными условиями по температуре (11). В качестве начальных условий по напряжениям задаются условия отсутствия перемещений в блоке $U_{i, j, k}^{0}=0, V_{i, j, k}^{0}=0, W_{i, j, k}^{0}=0$, а по температуре - условия (12) и (13).

Подпрограмма вычислений полей термоупругих напряжений была включена в цепочку программ, осуществляющих расчет всех этапов разрушения по схеме обмена информацией с другими подпрограммами [59]. В качестве выходных данных расчета рассмотрено поле максимальных напряжений $\sigma_{1}$, поскольку оно является необходимым (рис. 2,3). 


\section{Приведение результатов расчета к размерному виду}

При определении термоупругих напряжений надо знать в конечном счете распределение температур, что, в свою очередь, требует знания распределения тепловых источников, а это возможно лишь после нахождения потенциала электрического поля. Для этого рассмотрим случай электрода радиуса $r_{0}=0.02 \mathrm{~m}$, к которому приложено напряжение $V=10 \mathrm{kV}$, частота $f=5.28 \mathrm{MHz}$. Для гранита $\varepsilon^{\prime}=6$ и $\operatorname{tg} \delta=0.02$. Тогда $q_{0}=36.5 \mathrm{MW} / \mathrm{m}^{3}$ и $T_{0}=4896 \mathrm{~K}, T=\bar{T} T_{0}+T_{H}=440^{\circ} \mathrm{C}$. Введем размерную величину напряжений $\sigma_{0}=2740 \mathrm{MPa}$ при $E=70 \mathrm{GPa}$, $v=0.25, \alpha_{T}=6 \cdot 10^{-6} \mathrm{~K}^{-1}$, тогда размерное главное напряжение при $\bar{\sigma}=0.08$ равно $\sigma=\bar{\sigma} \sigma_{0}=219 \mathrm{MPa}$.

Таким образом, получено аналитическое решение задачи определения температурного поля (рис. 3) и поля напряжений в блоке породы при ВЧ нагреве двумя цилиндрическими электродами, расположенными в шпурах. При определении полей напряжений использована модель, не учитывающая влияния свободных поверхностей в шпуре.

Полученные численным моделированием картины распределения полей температур и термонапряжений показывают, что магистральная трещина будет развиваться с наружных граней блока и почти на уровне нижнего основания электрода. Второй максимум растягивающих напряжений наблюдается в верхней части блока, где также предполагается развитие магистральной трещины.

\section{Механизмы развития магистральных трещин}

Для методической отладки алгоритма была выбрана модельная задача о прорастании магистральной трещины между двумя шпурами в породе типа гранита. Шпуры радиусом $R_{\mathrm{sp}}=21 \mathrm{~mm}$ располагались на расстоянии $d=10 R_{\mathrm{sp}}$ перпендикулярно расчетной плоскости. Радиус шпура разбивался на 4 счетные ячейки. $\Delta_{x}=\Delta_{y}=R_{\mathrm{sp}}=5.25 \mathrm{~mm}$. При $l_{0}=0.146 \mathrm{~mm}$ характерному размеру ячейки соответствует трещина второго иерархического уровня $l_{2}=5.25 \mathrm{~mm}$, при этом $l_{1}=0.875 \mathrm{~mm}$. По линии шпуров задавалось симметричное, постоянное во времени распределение напряжений $\sigma_{y}$ нормальных линий шпуров. Три рассмотренных варианта распределения напряжений $\sigma_{y}$ приведены на рис. 4.

Расчеты проводились для следующих значений прочностных и термокинетических параметров породы: $\sigma_{\text {comp }}=120 \mathrm{MPa}, \sigma_{\text {tens }}=10 \mathrm{MPa}, v=0.3, U_{j}=10^{5} \mathrm{~J} / \mathrm{mol}$, $\gamma=9.375 \cdot 10^{-3} \mathrm{~m}^{3} / \mathrm{mol}, T=300 \mathrm{~K}$.

Результаты расчета распространения фронта разрушения породы от времени по линии шпуров представлены на рис. 5.

В рассматриваемой постановке задачи время установления поля напряжений полагается мгновенным. В связи

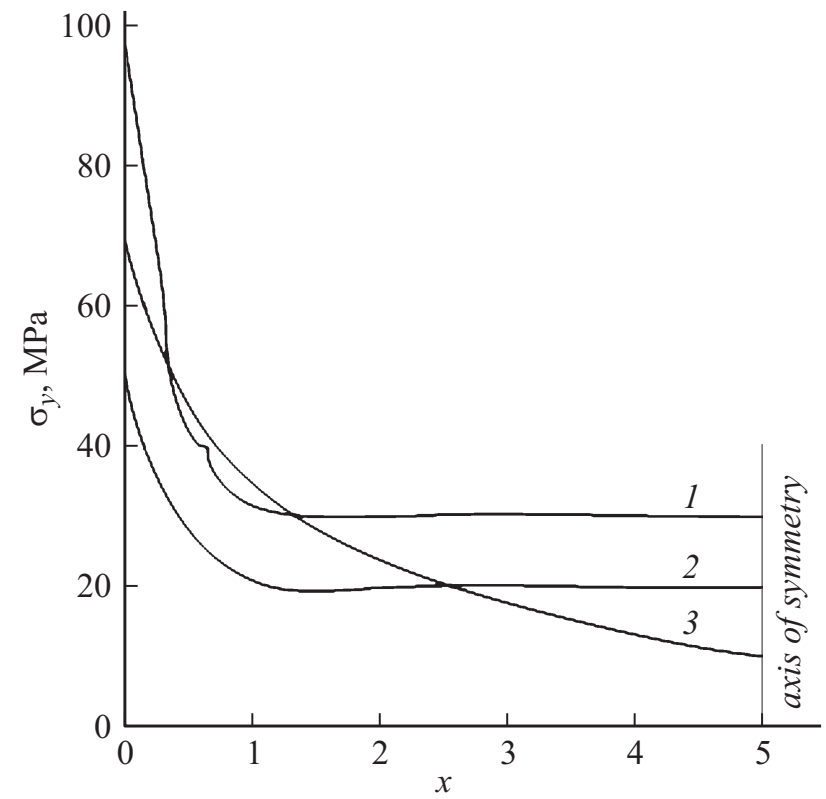

Рис. 4. Геометрия модельной задачи и три варианта распределения напряжений $\sigma_{y}$ по оси между шпурами. $x=(N) 5$ ячеек.

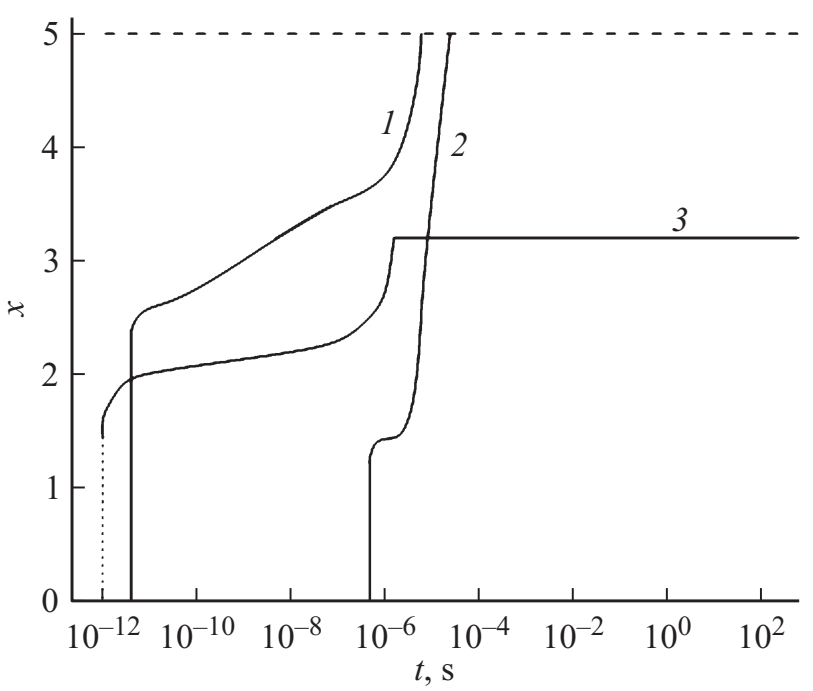

Рис. 5. Зависимость расстояния распространения фронта разрушения от времени для трех вариантов распределения напряжений $\sigma_{y}$ по оси между шпурами. $x=(N) 5$ ячеек.

с этим в случае нагрузки с распределением напряжений по линии, ограниченной расстоянием $x=2.25 R_{\mathrm{sp}}$ происходят зарождение, накопление и слияние микротрещин и трещин первого уровня. В результате образуются трещины второго уровня с характерным размером, равным характерному размеру счетной ячейки. Тем самым достигается разрушение породы в ячейках указанной области. Размер этой области соответствует уровню напряжений, равному пределу прочности на растяжение.

В более удаленных ячейках, где нагрузка падает ниже предела прочности на растяжение, скорость разрушения 
механизмом роста концентрации микротрещин падает, и начиная с моментов времени $t=10^{-6}$ s разрушение происходит механизмом прорастания магистральной трещины. В случае нагрузки с распределением напряжений по кривой 2 (рис. 4) характер разрушения сохраняется (кривая 2 на рис. 5). Поскольку нагружающие напряжения ниже, чем в первом случае, уменьшается размер области, разрушаемой в результате роста концентрации микротрещин, и увеличивается роль механизма магистральной трещины. В этих случаях магистральная трещина прорастает до плоскости симметрии, что обеспечивает разрушение породы между двумя шпурами с образованием магистральной трещины между ними.

Механизм разрушения ростом концентрации микротрещин обеспечивает разрушение породы вплоть до областей, в которых величина максимальных растягивающих напряжений становится равной $\sigma \approx 0.7\left[\sigma_{\text {tens }}\right]$, a механизм роста магистральной трещины - до уровня напряжений (рис. 4,5$) \sigma \approx 0.7\left[\sigma_{\text {tens }}\right]$, т.е. в областях, не отвечающих критерию максимальных растягивающих напряжений. Нагрузка с распределением напряжений по кривой 3 (рис. 4) отличается тем, что при $x=2.75 R_{\mathrm{sp}}$ напряжения становятся сжимающими. В этом случае разрушение вплоть до уровня нагрузок $\sigma \approx 0.7\left[\sigma_{\text {tens }}\right]$ сохраняет все указанные выше закономерности. Включающийся затем механизм роста магистральной трещины обеспечивает ее прорастание только лишь до границы области растягивающих напряжений, и разрушения области сжимающих напряжений не происходит даже при временах нагружения, превышающих $10^{3} \mathrm{~s}$ (кривая 3 на рис. 5). Отсюда следует, что границу области растягивающих напряжений можно трактовать как границу области, за которую разрушение, начавшееся в области больших растягивающих напряжений, проникнуть не может.

Теоретическое исследование комплекса этих процессов традиционными аналитическими методами представляет собой весьма сложную задачу [73-80].

В дальнейших работах будут изложены материалы исследования и подробно будет изложено развитие магистральной трещины в области растягивающих напряжений при применении метода акустико-эмиссионного прогнозирования механического разрушения и кинетическая модель микротрещинообразования твердых тел [81-91] с одновременной регистрацией электростатических полей [92-97].

\section{Выводы}

Научная значимость исследований состоит в разработке модели развития микро- и макротрещиноватости при воздействии электромагнитных полей с помощью ВЧ электродов.

Полученные численным моделированием картины распределения полей температур и термонапряжений показывают, что магистральная трещина будет развиваться с наружных граней блока и почти на уровне нижнего основания электрода. Второй максимум растягивающих напряжений наблюдается в верхней части блока, где также предполагается развитие магистральной трещины.

Предложена методика численного расчета развития магистральной трещины в одномерном поле растягивающих напряжений, сформированном в результате электромагнитного нагрева. Методика позволяет определить длину образующей трещины в зависимости от распределения растягивающих напряжений в породе и времени нагрева.

\section{Список литературы}

[1] Саломатов Вл.В., Пащенко С.Э., Сладков С.О., Саломатов В.В. // Инженерно-физический журн. 2016. Т. 89. № 1. C. 49.

[2] Meadows D.H., Meadows D.L., Rangers J., Beh-rens W.W. The Limits to Growths. NY: University Books, 1972. P. 70-77.

[3] Rhodes M. Introduction to particle technology, Monash University, Australia. 2nd Edition, John Wiley \& Sons Ltd, The Atrium, Southern Gate, Chichester, West Sussex PO19 8SQ, England, 2008.

[4] Singh Kedar Prasad, Kakati Mohan Chandra // Res. and Ind. 1994. Vol. 39. № 3. C. 198-201.

[5] Данилов О.С., Михеев В.А., Москаленко Т.В. // Горн. информ.-аналит. бюллетень. 2010. № 3. С. 203-208.

[6] Данилов О.С., Михеев В.А., Москаленко Т.В. // Изв. Самарского НЦ РАН. 2011. Т. 13. № 1 (5). С. 1264-1267.

[7] Marland S., Han B., Merchant A., Rowson N. // Fuel. 2000. Vol. 79. P. $1283-1288$.

[8] Marland S., Merchant A., Rowson N. // Fuel. 2001. Vol. 80. P. $1839-1849$.

[9] Данилов О.С., Михеев В.А., Москаленко Т.В. // Горн. информ.-аналит. бюллетень. Региональное приложение. Дальний Восток. 1. 2009. Вып. 4. С. 285-289.

[10] Lichao Ge, Yanwei Zhang, Zhihua Wang, Junhu Zhou, Kefa Cen // Energy Conversion and Management. 2013. Vol. 71. P. 84-91.

[11] Lester E., Kingman S. // Fuel. 2004. Vol. 83. P. 1941-1947.

[12] Красновский С.С. // Горный информ.-аналит. бюллетень. 1999. № 3. C. 163.

[13] Образцов А.П., Уваров А.П., Максименко А.Г. // Сб. Термомеханические методы разрушения горных пород. Киев: Наукова думка, 1976. С. 149.

[14] Автореф. канд. дис. Красновский С.С. Исследование взаимодействия электромагнитных полей ВЧ и СВЧ с горными породами для разработки способов и средств их разрушения: М., 1999. 46 с.

[15] Долголаптев А.В., Вороновский Ю.Д., Красновский С.С., Нистратов В.Ф., Образцов А.П. // Горный информ.аналит. бюллетень. 2000. Т. 10. С. 99-101.

[16] Mohindar S. // Department of Physics. West Virginia University, United States, 2011. P. 164-168.

[17] Пат. № 2514826. Способ СВЧ-градиентной активации угольного топлива с использованием защитной пленки / Пащенко С.Э., Алексеенко С.В., Пащенко С.С., Коляда В.В., Саломатов В.В. 2012. Бюл. № 13. 
[18] Старовойт А.Г., Малый Е.И., Чемеринский М.С. // Кокс и химия. 2010. № 9. С. 2-4.

[19] Lester E., Kingman S., Dodds C., Patrick J. // Fuel. 2006. Vol. 85. P. 2057-2063.

[20] Ruisànchez E., Arenillas A., Juàrez-Pèrez E.J., Menèndez J.A. // Fuel. 2012. Vol. 102. P. 65-71.

[21] Menèndez J.A., Arenillas A., Fidalgo B., Fernàndez Y., Zubizarreta L., Calvo E.G., Bermàdez J.M. // Fuel Proc. Technol. 2010. Vol. 91. P. 1-8.

[22] Menèndez J.A., Juàrez-Pérez E.J., Ruisànchez E., Bermàdez J.M., Arenillas A. // Carbon. 2010. Vol. 49. P. 346-349.

[23] Kanilo P.M., Kazantsev V.I., Rasyuk N.I., Schünemann K., Varviv D.M. // Fuel. 2003. Vol. 82. N 2. P. 187-193.

[24] Varviv D.M., Kazantsev V.I., Kanilo P.M., Rasyuk N.I., Schünemann K., Crytsayenko S.V. // Telecommunication and Radio Engineer. 2004. Vol. 61. N 8. P. 650-662.

[25] Hardgrove R.M. // Trans. Am. Soc. Mech. Eng. 1932. Vol. 54. P. 37-46.

[26] Серго E.E. Дробление, измельчение и грохочение полезных ископаемых: Учебник для вузов. М.: Недра, 1985.

[27] Austin L.G., Bagga P., Celik M. // Powder Technol. 1981. Vol. 28. P. 235-240.

[28] Kingman S.W., Rowson N.A. // Mineral Engineer. 1998. Vol. 11 (11). P. 1081-1088.

[29] Delibalta M.S., Toraman O.Y. // Energy Sci. Technol. 2012. Vol. 3. No 2. P. 46-49.

[30] Sahoo B.K., Dea S., Meikap B.C. // Fuel. Proc. Technol. 2011. Vol. 92. P. 1920-1928.

[31] Sahoo B.K., De S., Carsky M., Meikap B.C. // Ind. Eng. Chem. Res. 2010. Vol. 49. P. 3015-3021.

[32] Kingman $S$. Microwave pre-treatment of coal and coal blends to improve milling performance. BCURA Project B76, Final Report-January 2006.

[33] Диденко А.Н. СВЧ энергетика теория и практика. М.: Наука, 2003.

[34] Samanli S. // Fuel. 2011. Vol. 90. P. 659-664.

[35] Toraman O.Y. // Energy Sources. Part A. 2010. Vol. 32. P. $1794-1800$

[36] Delibalta M.S., Toraman O.Y. // Energy Sci. Technol. 2012. Vol. 3. N 2. P. $46-49$

[37] Altiner M., Yildirim M., Vapur H. Effects of Microwave and Conventional Drying Methods on Grinding Characteristics of Afsin-Elbistan Lignite. XIII Intern. Mineral Proc. Sympos. 2012. Bodrum, Turkey. P. 737-747.

[38] Marland S., Han B., Rowson N.A., Merchant A.J. // Acta Montanistica Slovaca Ročntk. 1998. Vol. 3. N 3. P. 351-355.

[39] Lester E., Kingman S. // Energy and Fuel. 2004. N 18. P. $140-147$.

[40] Lester E., Kingman S., Dodds C. // Fuel. 2005. Vol. 84. Pp. 423-427.

[41] Sahoo B.K., Dea S., Meikap B.C. // Fuel Proc. Technol. 2011. Vol. 92. P. 1920-1928.

[42] Kumar H., Lester E., Kingman S., Bourne R., Avila C., Jones A., Robinson J., Halleck P.M., Mathews J.P. // Intern. J. Coal Geology. 2011. Vol. 88. P. 75-82.

[43] Ruisànchez E., Arenillas A., Juàrez-Pèrez E.J., Menèndez J.A. // Fuel. 2012. Vol. 102. P. 65-71

[44] Takanashi T., Watanabe S. IEEE Trans. Nucl. Sci. 2001. Vol. 48. P. 950.

[45] Беляев А.А., Беляев А.Е., Ермолович И.Б. и др. // ЖТФ. 1998. T. 68. C. 49

[46] Редько Р.А., Будзуляк С.И., Корбутяк Д.В., Лоцько А.П., Вахняк Н.Д., Демчина Л.А., Калитчук С.М., Конакова Р.В., Миленин В.В., Быков Ю.В., Егоров С.В., Еремеев А.Г. // ФТП. 2015. Т. 49. № 7. С. 916
[47] Ключник А.В. // ЖТФ. 1992. Т. 69. С. 99.

[48] Корбутяк Д.В., Лоцько А.П., Вахняк Н.Д. и др. // ФТП. 2011. T. 45. C. 1175.

[49] Ермолович И.Б., Конакова Р.В., Миленин В.В. и др. // Физика и химия твердого тела. 2006. Т. 7. С. 763.

[50] Ермолович И.Б., Миленин Г.В., Миленин В.В. и др. // ЖТФ. 2007. Т. 77. С. 71.

[51] Журков С.Н., Куксенко В.С., Махмудов Х.Ф., Пономарев А.В. // ДАН. 1997. Т. 35. Вып. 4. С. 470-472.

[52] Куксенко В.С., Томилин Н.Г,, Махмудов Х.Ф., Бенин А.В. // Письма в ЖТФ. 2007. Т. 33. Вып. 2. С. 31-35.

[53] Куксенко В.С., Махмудов Х.Ф., Ильинов М.Д., Абдурахмонов 3.М. Вестник Инженерной школы Дальневосточного федерального ун-та. 2014. № 3 (20). С. 98-108.

[54] Куксенко В.С., Махмудов Х.Ф., Мансуров В.А., Султонов У., Рустамова М.3. // ФТПРПИ. 2009. № 4. С. 55-59.

[55] Махмудов Х.Ф. // Деформация и разрушение материалов. 2012. № 8. C. $41-45$.

[56] Махмудов Х.Ф., Куксенко В.С. // ФТТ. 2005. Т. 47. Вып. 5. C. $856-859$.

[57] Махмудов Х.Ф., Куксенко В.С., Томилин Н.Г., Бенин А.В. // Вестник Тамбовского ун-та. Сер. Естественные и технические науки. 2013. Т. 18. № 4-2. C. $1909-1910$.

[58] Менжулин М.Г., Махмудов Х.Ф., Куксенко В.С., Султонов У. // Вестник Тамбовского ун-та. Сер. Естественные и технические науки. 2013. Т. 18. № 4-2. С. 1667-1668.

[59] Менжулин М.Г., Махмудов Х.Ф., Щербаков И.П. LAMBERT Academic Publishing, 2014. C. 68.

[60] Менжулин М.Г., Махмудов Х.Ф., Щербаков И.П. Наука сегодня: теория, практика, инновации. Ростов-на-Дону, 2014. C. $159-187$.

[61] Махмудов Х.Ф., Менжсулин М.Г., Захарян М.В., Султонов У., Абдурахманов 3.М. // ЖТФ. 2015. Т. 85. Вып. 11. C. $79-85$.

[62] Менжулин М.Г., Махмудов Х.Ф., Томилин Н.Г., Бенин А.В., Султонов У., Абдурахманов 3.М. // Научное обозрение. 2015. № 24. С. 37-46.

[63] Rui Yao, Song Yi Liao, Chang Lu Dai, Yu Chen Liu, Xiao Yu Chen, Feng Zheng // International J. Heat and Mass Transfer. 1994. Vol. 37. N 6. P. 1013-1027.

[64] Li W., Ebadian M.A., White T.L., Grubb R.G., Foster D. // Chem. Engineer. Sci. 2011. Vol. 66. N 23. P. 5832-5851.

[65] Дмитриев А.П., Гончаров С.А. Термическое и комбинированное разрушение горных пород. М.: Недра, 1978.

[66] Менжсулин М.Г., Шишов А.Н., Серышев С.В. // ВНИМИ. 1995. C. $59-65$.

[67] Мисник Ю.М., Хоминский В.А. Разрушение горных пород электрофизическими методами Л.: ЛГИ, 1984. C. $65-85$.

[68] Красновский С.С., Образцов А.П. Расчет поля температур в породе и комплексной нагрузки генератора при высокочастотном контактном разрушении горных пород. Физика горных пород и процессов / Тез. докл. Всесоюзн. конф. ВУЗов с участием НИИ. М., 1971. С. 174-175.

[69] Красновский С.С. // Науч. сообщ. ИГД им. А.А. Скочинского. М., 1989. С. $101-108$

[70] Красновский С.С., Эпштейн Е.Ф., Образиов А.П. // Науч. сообщ. ИГД им. А.А. Скочинского. Вып. 54. М., 1968. С. $96-99$.

[71] Красновский С.С., Арш Э.И., Друкованный М.Ф. // Известия ДГИ. Т. 40. Днепропетровск, 1961. С. 124-130.

[72] Красновский С.С., Захаров Ю.Н. Новые возможности высокочастотного контактного способа разрушения горных пород. Тез. докл. Неделя горняка. М.: МГГУ, 1994. C. 193. 
[73] Литвиненко В.С., Богуславский Э.И., Коржсавых П.В. // Записки Горного института. 2012. Т. 195. С. 115-119.

[74] Завьялов А.Д. // ФТТ. 2005. Т. 47. Вып. 6. С. 1000-1008.

[75] Опарин В.Н., Востриков В.И., Усольцева О.М., Цой П.А., Семенов В.Н. // Физико-технические проблемы разработки полезных ископаемых. 2015. № 3. С. 180-191.

[76] Соболев Г.А., Веттегрень В.И., Ружич В.В., Киреенкова С.М., Смульская А.И., Мамалимов Р.И., Кулик В.Б. // Геофизические исследования. 2015. Т. 16. № 4. С. 5-14.

[77] Мельников Н.Н., Козырев А.А. // Горный информационно-аналит. бюллетень (Научно-технический журнал). Спец. выпуск 56. 2015. С. 7-23.

[78] Адушкин В.В., Кочарян Г.Г., Остапчук А.А. // ДАН. 2016. T. 467. № 1. C. 86.

[79] Веттегрень В.И., Куксенко В.С., Щербаков И.П. // ЖТФ. 2011. Т. 81. Вып. 4. С. 148.

[80] Веттегрень В.И., Куксенко В.С., Щербаков И.П. // ФТТ. 20012. T. 54. С. 1342.

[81] Nosov V.V. // Russian J. Nondestructive Testing. 2016. Vol. 52. N 7. P. 386-399.

[82] Куксенко В.С., Ляшков А.И., Савельев В.Н. // Дефектоскопия. 1980. № 6. С. 57-63.

[83] Ляшков А.И., Инжсеваткин И.Е., Савельев В.Н. // Дефектоскопия. 1980. № 6. С. 98-101.

[84] Носов В.В. // Дефектоскопия. 2014. № 12. С. 24-35.

[85] Носов В.В., Бураков И.Н. // Дефектоскопия. 2004. № 2. C. $53-61$.

[86] Носов В.В., Ельчанинов Г.С. // Дефектоскопия. 2011. № 12. C. 55-66.

[87] Носов В.В., Лаврин В.Г. // Дефектоскопия. 2012. № 3. C. $18-26$.

[88] Nosov V.V., Lachova T.N. // Russian J. Nondestructive Testing. 2012. Vol. 48. N 2. P. 75-84.

[89] Stanchits S., Burghardt J., Surdi A. // Rock. Mech. Rock. Eng. 2015. Vol. 48. P. 2513-2527.

[90] Lacidogna G., Carpinteri A., Manuello A., Durin G., Schiavi A., Niccolini G., Agosto A. // Strain. 2011. Vol. 47. (SUPPL. 2). P. 144-152.

[91] Nosov V.V., Potapov A.I. //Russian J. Nondestructive Testing. 2015. Vol. 51. N 1. P. 50-58.

[92] Lockner D.A., Byerlee J.D., Kuksenko V.S., Ponomarev A.V. // Pure Appl. Geophys. PAGEOPH. 1986. Vol. 124. N 3. P. $601-608$.

[93] Lockner D.A., Stanchits S.A. // J. Geophys. Research. B: Sol. Earth. 2002. Vol. 107. N 12. P. ETG 13-1-13-14.

[94] Soloviev S.P., Spivak A.A. // Izvestiya Phys. Sol. Earth. 2009. Vol. 45. N 4. P. 347-355.

[95] Махмудов Х.Ф. // ЖТФ. 2011. Т. 81. Вып. 1. С. 76.

[96] Куксенко В.С., Махмудов Х.Ф. // Письма в ЖТФ. 2004. Т. 30. Вып. 14. С. 82-88.

[97] Махмудов Х.Ф. // Современные наукоемкие технологии. 2015. № 12-3. C. 426-428. 